Exp. Anim. 67(4), 479-486, 2018

\title{
-Original-
}

\section{Increase in blood-brain barrier permeability does not directly induce neuronal death but may accelerate ischemic neuronal damage}

\author{
Chiemi OHMORI ${ }^{1)}$, Yusuke SAKAI ${ }^{1)}$, Yasuki MATANO ${ }^{1)}$, Yasuhiro SUZUKI ${ }^{2)}$, \\ Kazuo UMEMURA ${ }^{3)}$ and Nobuo NAGAI ${ }^{1)}$ \\ 1) Department of Animal Physiology, Division of Bioscience, Nagahama Institute of Bio-Science and Technology, \\ 1266 Tamura, Nagahama, Shiga 526-0829, Japan \\ 2) School of Pharmaceutical Sciences, Ohu University, 31-1 Tomita-cho Aza Sankaku-do, Koriyama, Fukushima \\ 963-8611, Japan \\ ${ }^{3)}$ Department of Pharmacology, Hamamatsu University School of Medicine, 1-20-1 Handayama, Higashi-ku, \\ Hamamatsu, Shizuoka 431-3192, Japan
}

\begin{abstract}
It is observed that the increase in blood-brain barrier (BBB) permeability (BBBP) is associated with ischemic stroke and thought to trigger neuronal damage and deteriorate ischemic infarction, even though there is no experimental proof. Here, we investigated the effect of BBBP increase on brain damage, using a combination of photochemically-induced thrombotic brain damage (PIT-BD) model, a focal brain ischemic model, and transient bilateral carotid artery occlusion model (CAO, a whole brain ischemic model), in mice. In PIT-BD, BBBP increased in the region surrounding the ischemic damage from $4 \mathrm{~h}$ till $24 \mathrm{~h}$ with a peak at $8 \mathrm{~h}$. On day 4 , the damaged did not expand to the region with BBBP increase in mice with PIT-BD alone or with 30 min CAO at $1 \mathrm{~h}$ before PIT-BD, but expanded in mice with 30 min CAO at $3.5 \mathrm{~h}$ after PIT-BD. This expansion was paralleled with the increase in the number of apoptotic cells. These findings indicate that increase in BBBP does not cause direct neuronal death, but it facilitates ischemic neuronal loss, which was attributed, at least partially, to acceleration of apoptotic cell death.
\end{abstract}

Key words: apoptosis, blood-brain barrier, ischemic stroke, neuronal death, permeability

\section{Introduction}

The permeability of brain vessels are restricted by the blood-brain barrier (BBB). It is known that the permeability of $\mathrm{BBB}$ is increased in the region surrounding ischemic area in both human patients [14] and experimental animal models [6, 13, 17]. It has been thought that the increase in BBB permeability (BBBP) is a deteriorative factor in ischemic stroke [1], although ex- perimental evidence for this hypothesis is scarce. This is resulted from the technical difficulty of separating the ischemic stress and increase in the BBBP, that is, BBBP increase was associated with ischemic stress caused by vessel occlusion in recent animal stroke models.

Here, we studied the effect of increase in the BBBP on ischemic neuronal damage by combination of a photochemically-induced thrombotic brain damage (PITBD) model [5] and common carotid occlusion model of

(Received 15 March 2018 / Accepted 2 May 2018 / Published online in J-STAGE 28 May 2018)

Address corresponding: N. Nagai, Department of Animal Physiology, Division of Animal Bioscience, Nagahama Institute of Bio-Science and Technology, 1266 Tamura, Nagahama, Shiga 526-0829, Japan

cc) $\$$ This is an open-access article distributed under the terms of the Creative Commons Attribution Non-Commercial No Derivatives (by-nc-nd) License <http://creativecommons.org/licenses/by-nc-nd/4.0/>. 

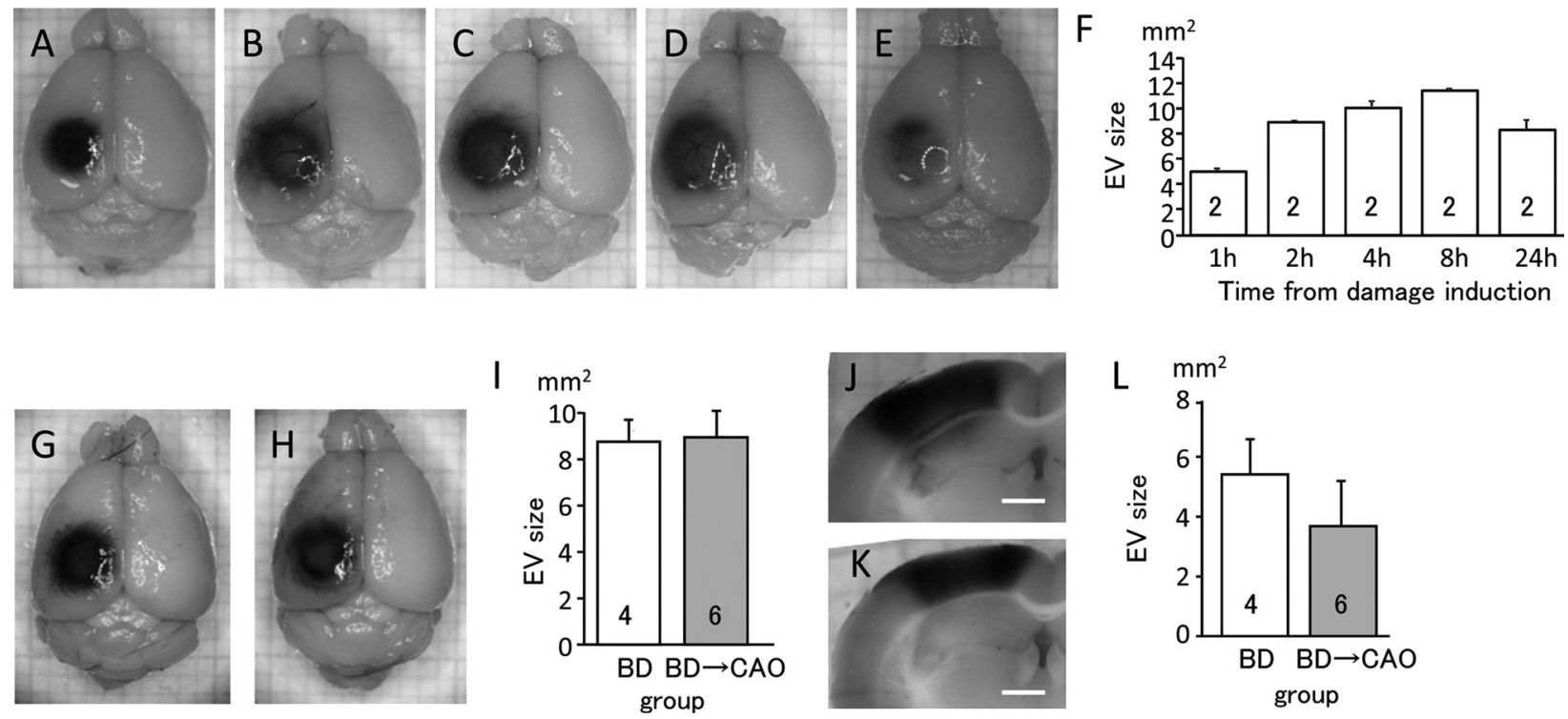

Fig. 1. Increase in the BBB permeability in the PIT-BD model. The increase in the BBB permeability was evaluated by extravasation of Evans Blue. Photographs of the brain surface at $1 \mathrm{~h}(\mathrm{~A}), 2 \mathrm{~h}(\mathrm{~B}), 4 \mathrm{~h}(\mathrm{C}), 8 \mathrm{~h}(\mathrm{D})$, and $24 \mathrm{~h}(\mathrm{E})$, in mice with brain damage alone. Quantification of the size of the region with the increase BBB permeability at each time (F). Photographs of the surface $(\mathrm{G}$ and $\mathrm{H}$ ) or sections at the center of the damage ( $\mathrm{J}$ and $\mathrm{K}$ ) in mice with PIT-BD alone ( $\mathrm{G}$ and $\mathrm{J}$ ) or with CAO after PIT-BD (H and G) at $4 \mathrm{~h}$. Quantification of the damage size on the surface (I) and the center section (L) with increased BBB permeability. Data represents mean and SD. The numbers in each column indicate the number of mice in each group. Bars indicate $1 \mathrm{~mm}$ in $\mathrm{J}$ and $\mathrm{K}$. Data represents mean and SD in F, I, and L.

whole brain ischemia condition by transient bilateral common carotid artery occlusion (CAO) model. In PITBD model, an ischemic brain damage was induced by thrombotic occlusion of cerebral blood vessels, via endothelial damage by a photochemical reaction in which singlet oxygen was synthesized by the infusion of photosensitive dye, Rose Bengal, and the subsequent illumination at a certain part of the cerebral cortex [15]. Since the damage size and region was strictly controlled by the light intensity and location of illumination, it is possible to trace sequential change of the damage in independent animals [5].

In the current experiments, we first found that BBBP was increased in the region surrounding the ischemic damage without neuronal death until $24 \mathrm{~h}$ after illumination in the PTI-BD model as shown in Fig. 1, indicating that the increase in BBBP alone did not cause neuronal damage. The BBBP increase was associated, probably not principally with the ischemic stress, but to the slight photochemical damage of the endothelial cells. In patients with ischemic stroke, however, BBBP increase is associated with ischemic damage of cerebral vasculature due to occlusion of the vessels. Therefore, we assume that the brain tissue with increased BBBP, in patients, was also subject to ischemic stress. In this report, we induced ischemic stress in the region of increased BBBP in the PIT-BD model, by combination of whole brain ischemia condition by transient bilateral common carotid artery occlusion (CAO) model.

\section{Materials and Methods}

The animal experiments were performed in accordance with the recommendations in the Guide for the Care and Use of Laboratory Animals, of the Japan Society for the Promotion of Science, and an ethical approval from the institutional rules for the care and use of laboratory animals of Nagahama Institute of BioScience and Technology (Approval No. 017).

C57BL/6 male mice (CLEA Japan, Tokyo, Japan), weighing $22-26 \mathrm{~g}$, were used. A focal ischemic brain damage was induced by PIT-BD model, as described previously [5]. Briefly, under isoflurane anaesthesia, the mice were placed on a heated pad maintained at $37^{\circ} \mathrm{C}$, a cannula was inserted in the jugular vein. The skin on the top of the head was incised, and an optic fibre (diameter, $1.0 \mathrm{~mm}$ ) was placed directly or through a filter (diameter, $0.7 \mathrm{~mm}$ ) at a position on the skull (bregma- 

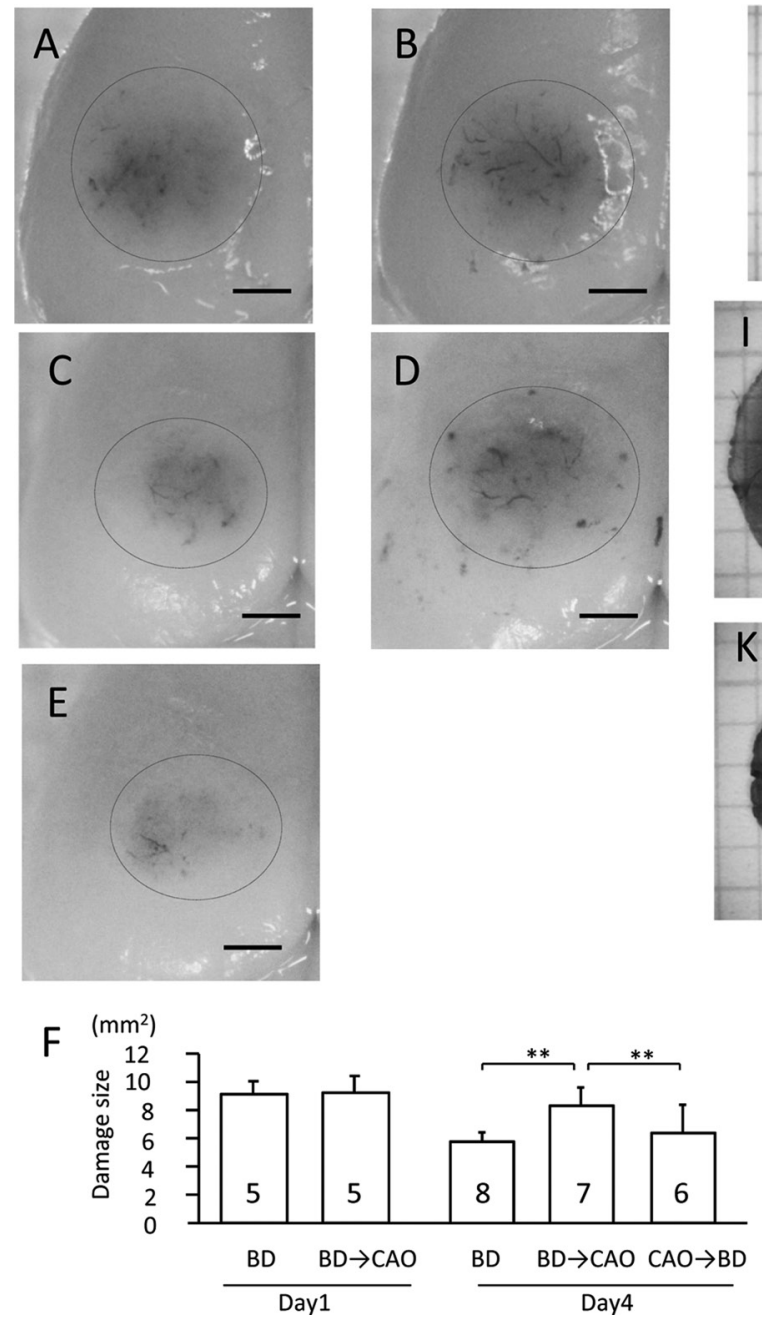
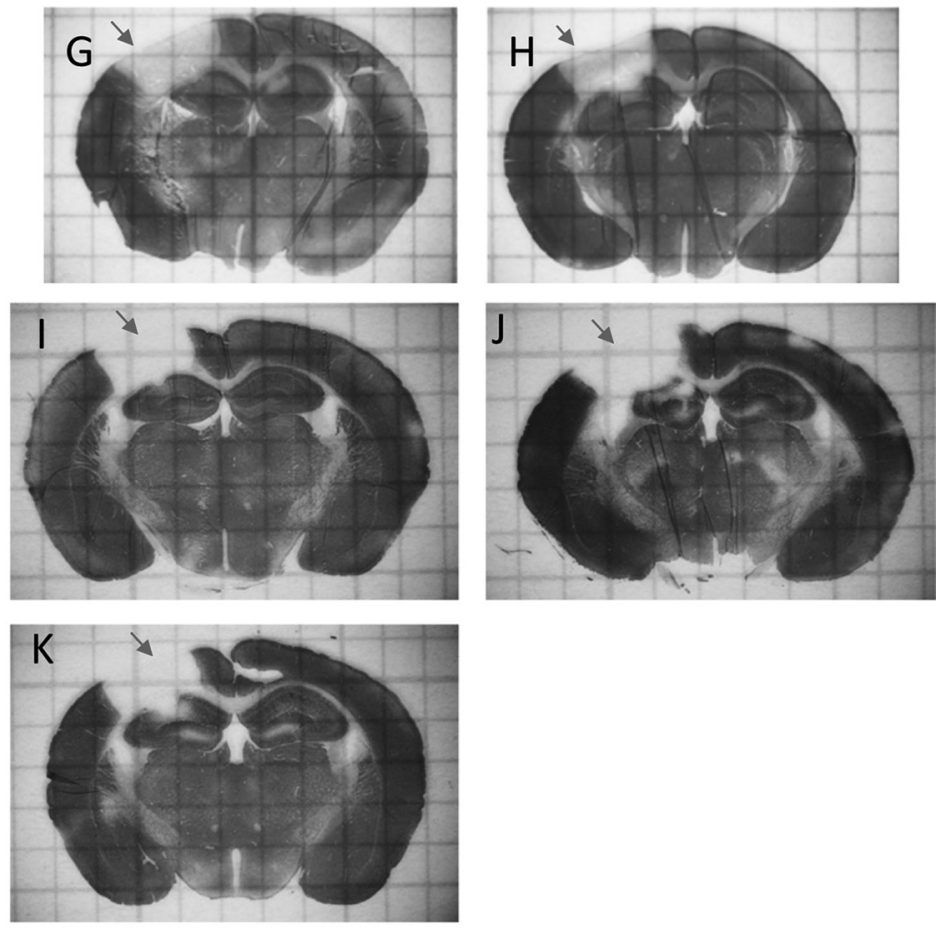

$\mathrm{L}$

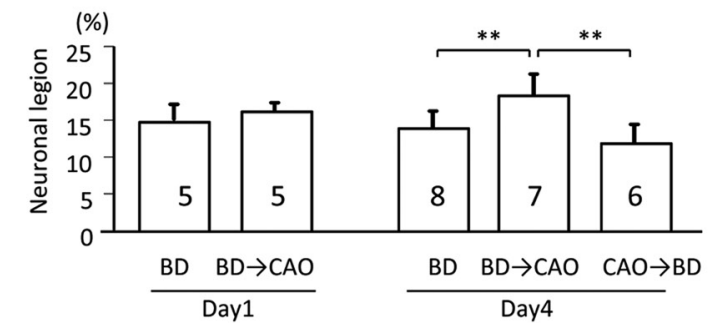

Fig. 2. Brain damage in the PIT-BD model combined with CAO. Photographs of the brain surface (A-E) or the MAP2 stained section at the middle of the damage $(\mathrm{G}-\mathrm{K})$ on day $1(\mathrm{~A}, \mathrm{~B}, \mathrm{G}, \mathrm{H})$ or on day $4(\mathrm{C}-\mathrm{E}, \mathrm{I}-\mathrm{K})$ in mice with PIT-BD alone $(\mathrm{A}, \mathrm{C}, \mathrm{G}, \mathrm{I})$, with CAO after PIT-BD (B, D, H, J), or with CAO before PIT-BD (E and K). The border of the damages on the brain surface are indicated by dotted lines (A-E). Quantification of the size of the damage, with 4-6 mice in each group, on day 1 (F) or day 4 (L). Data represents mean and SD. Bars in B, D, H K. The numbers in each column indicate the number of mice in each group. Bars in $\mathrm{A}-\mathrm{E}$ indicate $1 \mathrm{~mm}$. Grid in $\mathrm{G}-\mathrm{K}$ is $1 \mathrm{~mm}$ interval. Data represent mean and $\mathrm{SD}$. **: $P<0.01$.

lambda $=2.5 \mathrm{~mm}$, lateral $=1.5 \mathrm{~mm}$ to the left). Immediately after the infusion of $20 \mathrm{mg} / \mathrm{kg}$ Rose Bengal (Wako Pure Chemicals, Osaka, Japan) through the cannula was started, the area was illuminated with green light (wavelength, $540 \mathrm{~nm}$; illumination intensity, $4.7 \times$ $10^{-4} \mathrm{~W} / \mathrm{cm}^{2}$ ) for $10 \mathrm{~min}$ by using a light source (model L5178, Hamamatsu Photonics, Hamamatsu, Japan). After photoillumination, the skin was sterilely replaced and the cannula was withdrawn.

Whole brain ischemia was induced by transient occlusion of the bilateral common carotid artery (CCA). Mice, under isoflurane anesthesia, were placed on a heated pad, maintained at $37^{\circ} \mathrm{C}$, intubated intratrache- ally, while the anesthesia was maintained throughout the procedure using a ventilator (KN-58, NATSUME SEISAKUSHO, Tokyo, Japan). Then, the bilateral CCA was occluded over 30 min using clips [3], after which, the clips and trachea tube were dislodged, the hole of trachea was closed with a suture, and the skin was replaced. To prevent asphyxia by the material secreted in the trachea, $1 \mathrm{mg} / \mathrm{kg}$ of atropine (Mylan Pharma, Osaka, Japan) was intraperitoneally administered before the surgery.

After the surgery, animals were allowed to recover from the anesthesia, and were provided food and water ad libitum. A certain hours or days after the PIT-BD induction, the mice were anesthetized with an intraperi- 

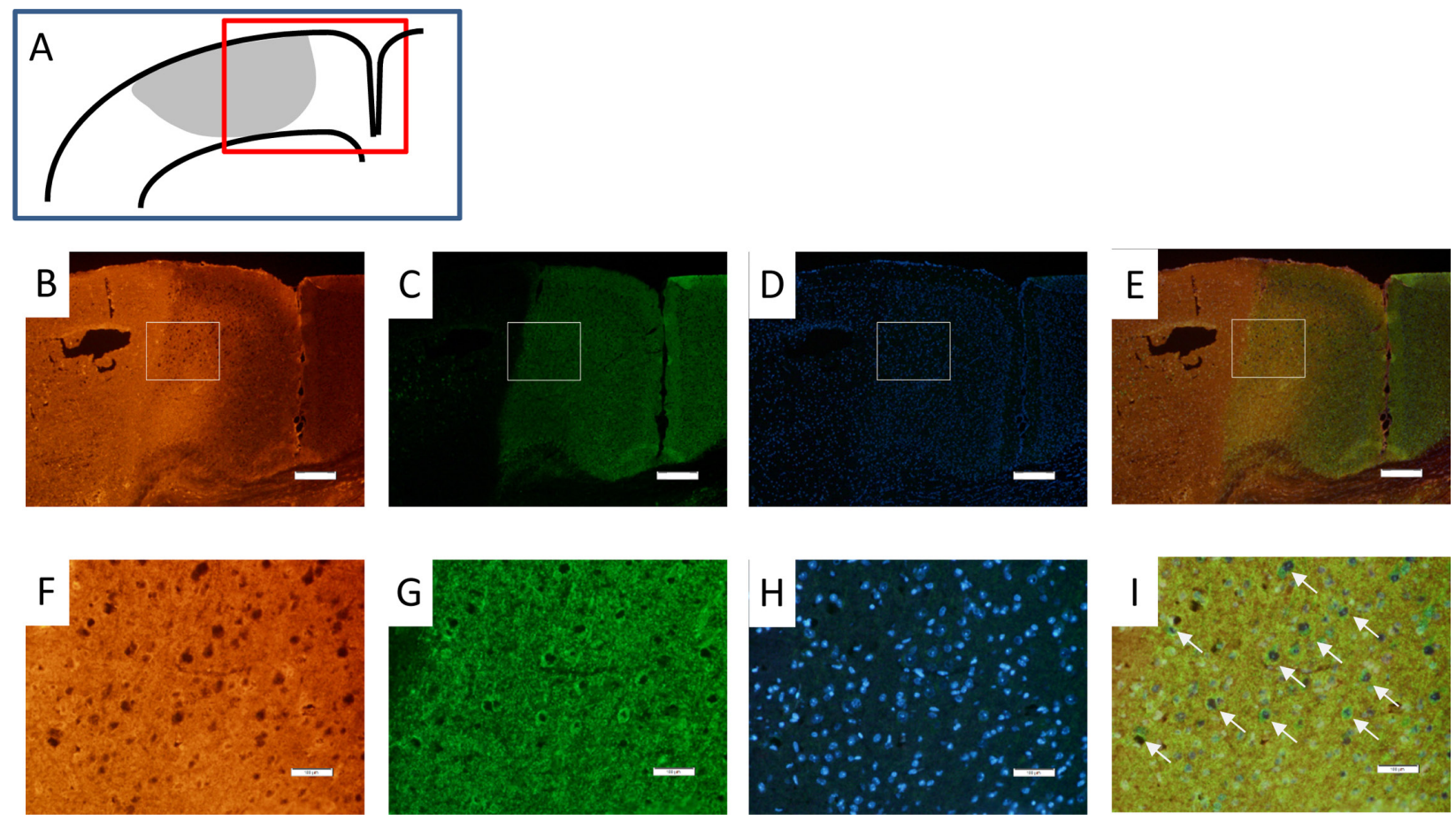

Fig. 3. Neurons in the region with BBB permeability increase. Photographs of the brain section on day 1 in mice with PIT-BD alone. A shows a cartoon of damaged section. Gray area indicates core region of damage where infarction was induced. The square indicates the region where photographed. Immunostaining with anti-rat IgG (B and F), or MAP2 (C and G), or staining with DAPI (D and H), Merged images of B-D, and F-H are shown in E and D, respectively. Magnified photographs of squares in B, $\mathrm{C}, \mathrm{D}$ and $\mathrm{E}$, are shown in F, G, H and I, respectively. Arrows in I indicates MAP2-positive neurons in rat IgG-positive region. Bar indicates $250 \mu \mathrm{m}$ in B-E, and $50 \mu \mathrm{m}$ in $\mathrm{F}-\mathrm{I}$.

toneal injection of pentobarbital, $70 \mathrm{mg} / \mathrm{kg}$, and perfused with $10 \mathrm{ml}$ of saline and $25 \mathrm{ml}$ of $4 \%$ paraformaldehyde for fixation. Then, the brains were dissected out and photographed.

To evaluate the increase in BBBP, extravasation of Evans Blue (EB) or rat IgG were evaluated as described elsewhere $[5,16]$. Briefly, mice were intravenously administered $200 \mathrm{mg} / \mathrm{kg}$ of $\mathrm{EB}$ or $5 \mathrm{mg} / \mathrm{kg}$ of rat $\mathrm{IgG}$ (Medical \& Biological Laboratories, Nagoya, Japan). Ninety minutes after injection, the mice were perfused with $50 \mathrm{ml}$ of saline. The damaged or EB-leaked region was measured, on the brain surface or on the coronal section, in the center of the damage. A good correlation was observed between the damage volume and either the area (in square millimeters) on the brain surface or the coronal section of the center of damage in this model [5]. For evaluation of rat IgG leakage, the brains were dissected out after paraformaldehyde fixation and used for immunohistochemistry, as described later.

After paraformaldehyde fixation, the brains were embedded in paraffin and the coronal $5-\mu \mathrm{m}$-thick sections in the center of the damage were obtained. These sections were immunostained as described elsewhere [5, 16]. For rat IgG staining, sections were treated with goat anti rat-IgG peroxidase conjugate (Abcam, Tokyo, Japan). For staining of microtubule associated protein 2 (MAP2), a marker of neurons, or F4/80, a marker of reactive microglia, sections were treated with either anti-MAP2 antibody (Abcam, Tokyo, Japan) or anti-F4/80 antibody (Abcam, Tokyo, Japan), and subsequently an appropriate secondary antibody conjugated with Fluorosein, Cy3, or peroxidase. For Fluorosein and $\mathrm{Cy} 3$, immunoreactivity was observed by a fluorescence microscopy after counterstaining with 4',6-Diamidino-2-phenylindole dihydrochloride (DAPI), For peroxidase, immunoreactivity was visualized using diaminobenzidine coloration. The size of both BBBP increased region and the neuronal lesion were measured as the area of Rat IgG-positive and MAP2-negative against the area of the ipsilateral hemisphere in the sections, respectively. Apoptotic cell numbers were also visualized by terminal deoxynucleotidyl transferase dUTP nick-end labeling (TUNEL) assay with 

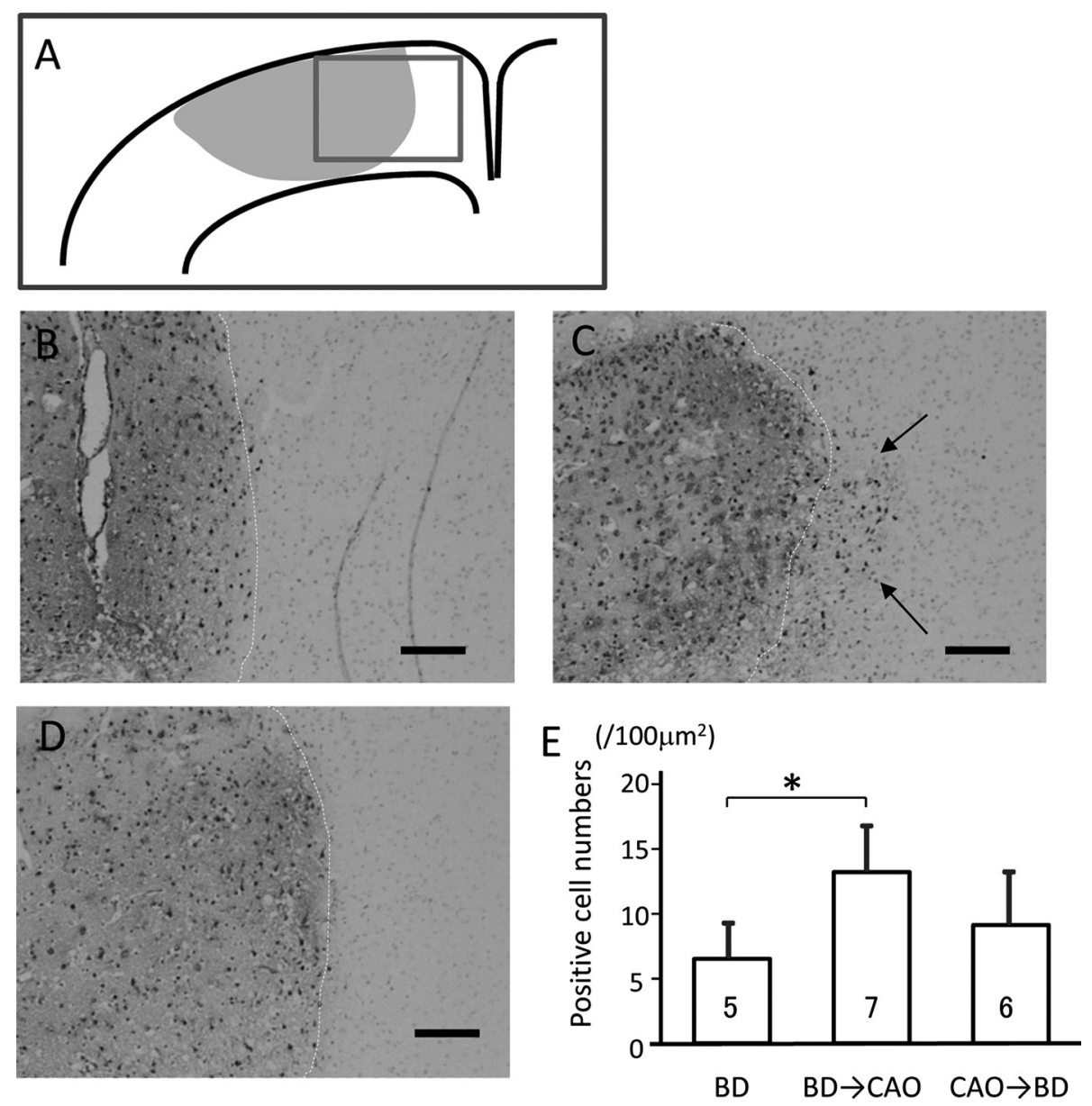

Fig. 4. TUNEL-stained brain sections. Cells death by apoptosis was indicated by TUNEL-staining at the medial border of the ischemic damage shown by a square in A. Photographs of TUNEL-stained coronal brain sections of mice with PIT-BD alone (B), with CAO after PIT-BD (C), or with CAO before PIT-BD (D) on day 4. Border of damages are indicated by dotted line in B-D. Arrows indicate TUNEL-positive cells present outside the ischemic damage. Bars indicate $50 \mu \mathrm{m}$. The numbers of TUNEL-positive cells in the region with both, 100 $\mu \mathrm{m}$ width inside and outside, from the medial border of the damage were shown in E. Data represent mean and SD. The number in each column indicates the number of mice in each group. *: $P<0.05$.

the In Situ Cell Death Detection Kit (Roche Diagnositics, Tokyo, Japan). The number of TUNEL-positive cells at the region, in both $100-\mu \mathrm{m}$ width inside and outside from the medial edge of the damage (Fig. 4A), were measured.

Statistical analysis was performed using ANOVA, followed by Dunnett's test for post-hoc analysis, or Student's $t$-test for comparison between groups. $P$-values less than 0.05 were considered significant.

\section{Results}

In the PIT-BD model, BBBP increased indicated by EB leakage was observed in the region surrounding the ischemic brain damage at $1 \mathrm{~h}$ after illumination, which expanded till $8 \mathrm{~h}$, and reduced at $24 \mathrm{~h}$ to the extent comparable to that at $2 \mathrm{~h}$ (Fig. 1A-F). This increase in BBBP was in line with previous observations $[5,13]$. Based on the findings, we chose to apply CAO at $3.5 \mathrm{~h}$ after illumination, over $30 \mathrm{~min}$, in the current experiments. To compare the additive effect of CAO on PIT-BD, we also evaluated in another condition in which $\mathrm{CAO}$ was induced $1 \mathrm{~h}$ before PIT-BD, over $30 \mathrm{~min}$. We then studied the effect of CAO on BBBP increase by PIT-BD. The size of BBBP-increasing region was comparable in mice with or without $\mathrm{CAO}$ after PIT-BD, which were measured both on the surface (Fig, $1 \mathrm{G}-\mathrm{I}, P=0.8736$ ) and in 
the section from the center of damage (Fig, $1 \mathrm{~J}-\mathrm{L}$, $P=0.7014)$ at $4 \mathrm{~h}$.

Next, we studied the damage size on day 1 and day 4 . These experiments were performed independently from the experiment for evaluating the BBBP-increasing region shown in Fig. 1. On day 1, the damage size on the surface of the brain in mice without CAO was comparable with those with CAO after PIT-BD $(P=0.9876)$, whereas the damage size on day 4 in mice with $\mathrm{CAO}$ after PIT-BD was significantly larger than in mice without $\mathrm{CAO}(P=0.0017)$ or with CAO before PIT-BD $(P=0.0204)$ (Fig. 2A-F). The time-dependent reduction of damage size in the PIT-BD model was also in line with previous observations [5]. We also assessed the size of neuronal lesion in the section from the center of damage. The lesion size in mice with CAO after PIT-BD was comparable with those with CAO before PIT-BD or without $\mathrm{CAO}$ on day 1 , whereas the lesion size was significantly larger in mice with CAO after PIT-BD than in mice without $\mathrm{CAO}(P=0.0073)$ or with $\mathrm{CAO}$ before PIT$\mathrm{BD}(P=0.0018)$ on day 4 (Fig. $2 \mathrm{G}-\mathrm{L})$.

We also evaluated BBBP and neuronal viability using histological analysis. On day 1, extravasation of rat IgG, which was injected intravenously, was observed in the region surrounding ischemic damage in the PIT-BD model (Fig. 3B and F). At the core region where infarction was induced, only MAP-2 negative died cells distributed (Fig. 3C and E), whereas a majority of the neurons in the rat $\mathrm{IgG}$ positive region at the surroundings of the damage were viable (Fig. 3C, E, G, I).

The involvement of apoptosis on the expansion of damage were studied by TUNEL staining. The TUNELpositive cells at the medial border of the damage, where BBBP was increased, existed more remarkable in mice with CAO after PIT-BD than in those without CAO, whereas it was comparable between mice with $\mathrm{CAO}$ before PIT-BD and without CAO (Fig. 4A-D). Practically, the number of TUNEL-positive cells at the medial border was significantly higher in mice with $\mathrm{CAO}$ after PIT-BD than in mice without $\mathrm{CAO}$ or with $\mathrm{CAO}$ before PIT-BD (Fig. 4E).

To evaluate the effect of damage associated molecular patterns (DAMPs), we compared the activation of microglia at the surrounding region of the damage. The number of the F4/80-positive reactive microglia was comparable between mice with PIT-BD alone and CAO after PIT-BD (Fig. 5).

\section{Discussion}

Since BBBP is associated with the ischemic stress which was induced not only vessels but also the surrounding neurons, it is not possible to evaluate the effect of BBBP increase and ischemic stress separately in general ischemic stroke models. In the present study, we studied the effect of BBBP increase and ischemic stress by combination of the PIT-BD model and the CAO model. In the PIT model, BBB was damage by singletoxygen induced by photochemical reaction at lumen of the vessel [15]. In core region of damage, vessels are occluded just after illumination. But surrounding region where BBBP increased with several hours delay must likely is resulted from the weak damage of endothelial cells. Because of short half-life of singlet-oxygen, the damage resulted in BBBP increase is likely limited at the endothelial cells, and not expanded to neurons. Practically, neurons in the region where BBBP increased were alive till day 4 . Therefore, we considered that BBBP increase was induced separately from ischemic stress in the PIT-BD model. On the other hand, whole brain ischemia can be induced with arbitrary duration by $\mathrm{CAO}$ model [7]. Therefore, we succeeded to evaluate the effect of BBBP increase and ischemic stress separately by their combination.

In the PIT-BD model, BBBP was increased inside of the damage and at the surrounding region from $2 \mathrm{~h}$ and prolonged at $24 \mathrm{~h}$. Taken together that neurons at the surrounding region where BBBP increased were still alive at $24 \mathrm{~h}$, it is indicated that BBBP increase alone was not deteriorative for neurons. In the present study, BBBP was evaluated by either leakage of EB or rat IgG. EB makes complex with plasma albumin [12]; hence, EB leakage indicates an increase in extravasation of not only small, but also large molecules like proteins. The leakage of rat IgG also supports it. Considering that several proteins and small molecules in plasma have toxic effects on neurons $[4,11]$, the BBB is thought to provide a barrier to separate these harmful compounds in circulating blood from brain parenchyma and its disruption is likely results in a deteriorative effect on the neurons. However, our results showed that extravasation of these plasma molecules via BBBP increase are not toxic for neurons.

Both size of the damage and neuronal lesion in mice with $\mathrm{CAO}$ after PIT-BD were significantly larger than in mice without $\mathrm{CAO}$ on day 4 , whereas, they were com- 

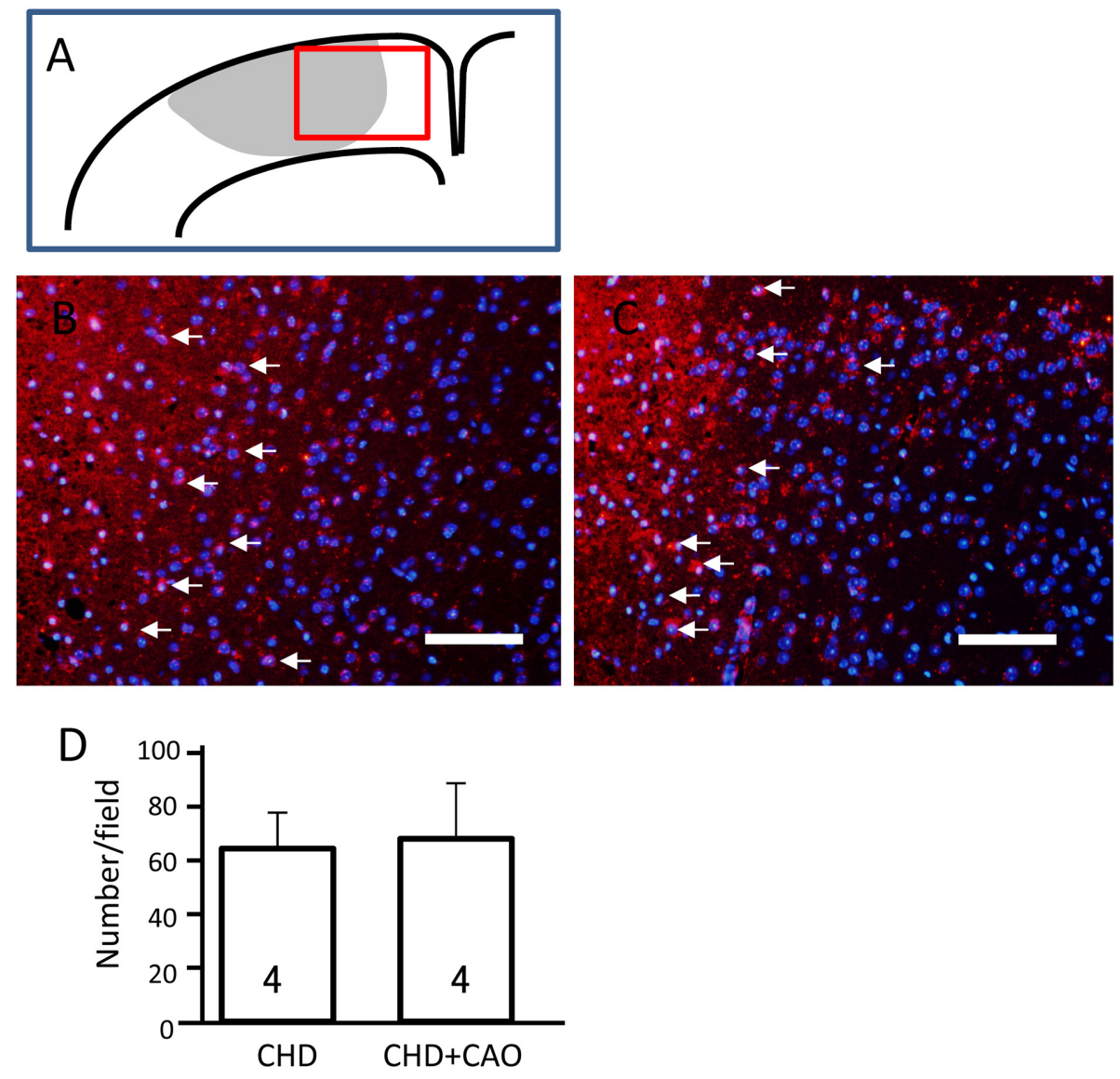

Fig. 5. F4/80 immunoreactivity in brain sections. Active microglia at the medial border of the ischemic damage of brain section shown by a square in A. Photographs of brain section of mice with PIT-BD alone (B) and CAO after PIT-BD (C). Red: F4/80 immunoreactivity, Blue: nuclear staining by DAPI. Bar indicates $50 \mu \mathrm{m}$. The numbers of F4/80-positive cell in a field were shown in D. D Data represent mean and SD. The number in each column indicates the number in each group.

parable on day 1 . These findings demonstrate that ischemic stress after BBBP increase accelerated neuronal loss from day 1 to day 4, although it did not affect neuronal viability within $24 \mathrm{~h}$. Considering that $\mathrm{CAO}$ before PIT-BD did not accelerate neuronal loss, the increased damage was not due to simple integration of the individual damages by BBBP increase and ischemic stress but enhancement of neuronal vulnerability against ischemic stress by BBBP increase. The leakage of plasma molecules as discussed above might be resulted in the acceleration of neuronal vulnerability.

In addition, the number of TUNEL positive cells at the peripheral region of the brain damage was significantly increased in mice with CAO after PIT-BD than PIT-BD alone or CAO before PIT-BD, indicating that the increase in damage size by subsequent whole brain ischemia was, at least partially, resulted in the acceleration of apoptotic neuronal loss. Recent studies indicate a possibility that the dysfunction of glutamate uptake by transporter in astrocytes and subsequent increase in glutamate toxicity is involved in the apoptotic neuronal death [10]. Since concentration of glutamate in plasma is higher $(40-60 \mu \mathrm{g} / \mathrm{ml})$ than that in brain parenchyma/ cerebrospinal fluid (1-10 $\mu \mathrm{g} / \mathrm{ml})$ [8], the increase in the BBBP may accelerate the apoptosis by glutamate toxicity.

We also assessed the involvement of DAMPs on BBBP increase. It is known that damaged cells release DAMPs, which cause accumulation and stimulation of leukocytes [9], and immune response is an exacerbating factor of ischemic stroke [2]. The number of reactive microglia at the surrounding region of the damage was comparable 
between mice with PIT-BD alone and CAO after PIT-BD $(P=0.7695)$. These findings indicate that the effect of DAMPs released from the damage was not altered by $\mathrm{CAO}$ via acceleration of immune response, even though a possibility which DAMPs release from core region increased the vulnerability of neurons directly at the surrounding region is still remained.

In conclusion, it was found that the BBBP increase alone did not cause neuronal death in the surrounding region of ischemic damage. Furthermore, whole brain ischemia by $\mathrm{CAO}$ not before but after BBBP increase by PIT-BD accelerated neuronal loss. These findings indicate that BBBP increase does not cause neuronal death directly, but may facilitates neuronal loss caused by ischemic stress, which was attributed, at least partially, to acceleration of apoptotic cell death.

\section{Acknowledgments}

This study was supported, Grant-in-Aid for Scientific Research (C) (grant 15K08194) and MEXT-Supported Program for the Strategic Research Foundation at Private Universities (grant 1201037) by the Ministry of Education, Culture, Sports, Science and Technology.

\section{References}

1. Bektas, H., Wu, T.C., Kasam, M., Harun, N., Sitton, C.W., Grotta, J.C., and Savitz, S.I. 2010. Increased blood-brain barrier permeability on perfusion CT might predict malignant middle cerebral artery infarction. Stroke 41: 2539-2544. [Medline] [CrossRef]

2. Chopp, M., Zhang, R.L., Chen, H., Li, Y., Jiang, N., and Rusche, J.R. 1994. Postischemic administration of an antiMac-1 antibody reduces ischemic cell damage after transient middle cerebral artery occlusion in rats. Stroke 25: 869-875, discussion 875-876. [Medline] [CrossRef]

3. Lee, S.R., Tsuji, K., Lee, S.R., and Lo, E.H. 2004. Role of matrix metalloproteinases in delayed neuronal damage after transient global cerebral ischemia. J. Neurosci. 24: 671-678. [Medline] [CrossRef]

4. Mhatre, M., Nguyen, A., Kashani, S., Pham, T., Adesina, A., and Grammas, P. 2004. Thrombin, a mediator of neurotoxicity and memory impairment. Neurobiol. Aging 25: 783-793. [Medline] [CrossRef]

5. Nagai, N., Kawao, N., Okada, K., Ishida, C., Okumoto, K., Ueshima, S., Suzuki, Y., Umemura, K., and Matsuo, O. 2010. Initial brain lesion size affects the extent of subsequent pathophysiological responses. Brain Res. 1322: 109-117.
[Medline] [CrossRef]

6. Nagai, N., Suzuki, Y., Van Hoef, B., Lijnen, H.R., and Collen, D. 2005. Effects of plasminogen activator inhibitor-1 on ischemic brain injury in permanent and thrombotic middle cerebral artery occlusion models in mice. J. Thromb. Haemost. 3: 1379-1384. [Medline] [CrossRef]

7. Nagai, N., Vanlinthout, I., and Collen, D. 1999. Comparative effects of tissue plasminogen activator, streptokinase, and staphylokinase on cerebral ischemic infarction and pulmonary clot lysis in hamster models. Circulation 100: 25412546. [Medline] [CrossRef]

8. O’Kane, R.L., Martínez-López, I., DeJoseph, M.R., Viña, J.R., and Hawkins, R.A. 1999. $\mathrm{Na}(+)$-dependent glutamate transporters (EAAT1, EAAT2, and EAAT3) of the bloodbrain barrier. A mechanism for glutamate removal. J. Biol. Chem. 274: 31891-31895. [Medline] [CrossRef]

9. Ooboshi, H. and Shichita, T. 2016. [DAMPs (damage-associated molecular patterns) and inflammation]. Nihon Rinsho 74: 573-578. (in Japanese) [Medline]

10. Ouyang, Y.B., Voloboueva, L.A., Xu, L.J., and Giffard, R.G. 2007. Selective dysfunction of hippocampal CA1 astrocytes contributes to delayed neuronal damage after transient forebrain ischemia. J. Neurosci. 27: 4253-4260. [Medline] [CrossRef]

11. Patel, J.P. and Frey, B.N. 2015. Disruption in the BloodBrain Barrier: The Missing Link between Brain and Body Inflammation in Bipolar Disorder? Neural Plast. 2015: 708306. [Medline] [CrossRef]

12. Patterson, C.E., Rhoades, R.A., and Garcia, J.G. 1992. Evans blue dye as a marker of albumin clearance in cultured endothelial monolayer and isolated lung. J. Appl. Physiol. 72: 865-873. [Medline] [CrossRef]

13. Suzuki, Y., Nagai, N., Yamakawa, K., Kawakami, J., Lijnen, H.R., and Umemura, K. 2009. Tissue-type plasminogen activator (t-PA) induces stromelysin-1 (MMP-3) in endothelial cells through activation of lipoprotein receptor-related protein. Blood 114: 3352-3358. [Medline] [CrossRef]

14. The NINDS t-PA Stroke Study Group 1997. Intracerebral hemorrhage after intravenous t-PA therapy for ischemic stroke. The NINDS t-PA Stroke Study Group. Stroke 28: 2109-2118. [Medline] [CrossRef]

15. Watson, B.D., Dietrich, W.D., Busto, R., Wachtel, M.S., and Ginsberg, M.D. 1985. Induction of reproducible brain infarction by photochemically initiated thrombosis. Ann. Neurol. 17: 497-504. [Medline] [CrossRef]

16. Yano, M., Kawao, N., Tamura, Y., Okada, K., Ueshima, S., Nagai, N., and Matsuo, O. 2011. Spatiotemporal differences in vascular permeability after ischaemic brain damage. $\mathrm{Neu}$ roreport 22: 424-427. [Medline] [CrossRef]

17. Yepes, M., Sandkvist, M., Moore, E.G., Bugge, T.H., Strickland, D.K., and Lawrence, D.A. 2003. Tissue-type plasminogen activator induces opening of the blood-brain barrier via the LDL receptor-related protein. J. Clin. Invest. 112: 15331540. [Medline] [CrossRef] 